\title{
STAR FORMATION EFFICIENCY IN THE BARRED SPIRAL GALAXY NGC 4303
}

\author{
Rieko Momose ${ }^{1,2}$, Sachiko K. OKumura ${ }^{2}$, Jin Koda ${ }^{3,4}$, and Tsuyoshi Sawada ${ }^{2}$ \\ ${ }^{1}$ Department of Astronomy, University of Tokyo, Hongo, Bunkyo-ku, Tokyo 113-0033, Japan; momo.s.rieko@nao.ac.jp \\ ${ }^{2}$ National Astronomical Observatory of Japan, Mitaka, Tokyo 181-8588, Japan; sokumura@nro.nao.ac.jp, tsawada@alma.cl \\ ${ }^{3}$ Department of Physics and Astronomy, Stony Brook University, Stony Brook, NY 11794-3800, USA; Jin.Koda@stonybrook.edu \\ ${ }^{4}$ California Institute of Technology, MS 105-24, Pasadena, CA 91125, USA \\ Received 2010 March 28; accepted 2010 July 22; published 2010 August 27
}

\begin{abstract}
We present new ${ }^{12} \mathrm{CO}(J=1-0)$ observations of the barred galaxy NGC 4303 using the Nobeyama $45 \mathrm{~m}$ telescope (NRO45) and the Combined Array for Research in Millimeter-wave Astronomy (CARMA). The H $\alpha$ images of barred spiral galaxies often show active star formation in spiral arms, but less so in bars. We quantify the difference by measuring star formation rate (SFR) and star formation efficiency (SFE) at a scale where local star formation is spatially resolved. Our CO map covers the central 2'.3 region of the galaxy; the combination of NRO45 and CARMA provides a high fidelity image, enabling accurate measurements of molecular gas surface density. We find that SFR and SFE are twice as high in the spiral arms as in the bar. We discuss this difference in the context of the Kennicutt-Schmidt (KS) law, which indicates a constant SFR at a given gas surface density. The KS law breaks down at our native resolution $(\sim 250 \mathrm{pc}$ ), and substantial smoothing (to $500 \mathrm{pc}$ ) is necessary to reproduce the KS law, although with greater scatter.
\end{abstract}

Key words: galaxies: individual (NGC 4303) - galaxies: ISM - galaxies: spiral - ISM: molecules - radio lines: galaxies

\section{INTRODUCTION}

Barred galaxies often reveal a striking difference in star formation activity between the bar and outer spiral arms (e.g., Downes et al. 1996; Sheth et al. 2002). Most star-forming regions ( $\mathrm{H} \alpha$ emission) are associated with the outer spiral arms rather than the bar, even when both host a similar amount of molecular gas.

The difference is often attributed to strong shear motions along the bar, by which giant molecular clouds (GMCs) could be pulled apart and disrupted before the ignition of star formation (e.g., Downes et al. 1996; Schinnerer et al. 2002). On the other hand, according to Koda \& Sofue (2006), the observed velocity gradient in a bar is not large enough to shred gravitationally bound GMCs.

Unfortunately, previous studies suffered from large uncertainties in measurements of gas surface density, due to the poor image quality (fidelity) of interferometer observations, or to low sensitivity and resolution even when the observations from a single-dish telescope were included. Interferometers have an intrinsic missing flux problem and cannot measure the gas surface density accurately. Most interferometric studies covered only a central bar region including the nucleus and did not extend to outer spiral arms, because of the limited field of view. Their studies concentrated on nuclear gas condensations and the gas fueling galactic centers (e.g., Sakamoto et al. 1999a) and did not consider the difference between the bar and outer spiral arms. At low resolution, the velocity gradients and gas surface densities appear similar in the inner bar and outer spiral arms, perhaps because of beam dilution (e.g., Kuno et al. 2007).

We quantitatively study the star formation rate (SFR) and star formation efficiency ( $\mathrm{SFE}=\mathrm{SFR} / M_{\mathrm{H}_{2}}$; the ratio of the SFR to the molecular gas mass) at a high resolution over the disk of the barred galaxy NGC 4303, using new molecular gas data at high quality. We capitalize on the recent development of the Combined Array for Research in Millimeter-wave Astronomy (CARMA) interferometer, and the 25 beam receiver (BEARS; Sunada et al. 2000) and on-the-fly (OTF) mapping technique
(Sawada et al. 2008) at the Nobeyama $45 \mathrm{~m}$ radio telescope (NRO45). The combination of CARMA and NRO45 solves the missing flux problem, providing the best image fidelity and quality and enabling accurate measurement of gas surface density. Our measurement is based on the fundamental transition $J=1-0$ of CO, which has often been used to derive the gas surface density (e.g., Scoville \& Sanders 1987; Young \& Scoville 1991). We occasionally refer to "molecular gas" as "gas" for simplicity in the rest of the paper.

We also investigate the star formation activity in the context of the Kennicutt-Schmidt (KS) law (Schmidt 1959; Kennicutt 1998). The KS law is the observational correlation between the area-averaged SFR $\left(\Sigma_{\mathrm{SFR}}\left[M_{\odot} \mathrm{yr}^{-1} \mathrm{kpc}^{-2}\right]\right)$ and gas surface density $\left(\Sigma_{\mathrm{H}_{2}}\left[M_{\odot} \mathrm{pc}^{-2}\right]\right)$ as a power law: $\Sigma_{\mathrm{SFR}} \propto \Sigma_{\mathrm{H}_{2}}^{N}$. This correlation of Kennicutt (1998) spans a wide range, five orders of magnitude in $\Sigma_{\mathrm{H}_{2}}$ and seven orders of magnitude in $\Sigma_{\mathrm{SFR}}$, with $N=1.4 \pm 0.15$ using a sample of 61 nearby spiral and 36 starburst galaxies.

Most recent studies concentrate on the KS law at high spatial resolutions and use local gas and SFR densities rather than the averages over galactic disks. The power-law index is a topic of interest, since it could indicate the physical origin of the correlation. A linear correlation, $N=1$, would indicate that the SFE is a constant over a large range of gas surface density; in other words, the mere presence of the gas is sufficient for star formation. On the other hand, a super-linear correlation, $N \neq 1$, may suggest that additional conditions or trigger mechanisms, such as gas dynamics, also play critical roles. Wong \& Blitz (2002) averaged their data azimuthally as a function of galactic radius for seven gas-rich galaxies and obtained $N=1.1$ and 1.7 (for two different extinction models). Kennicutt et al. (2007) used the locally averaged parameters $(520 \mathrm{pc}$ aperture) around $\mathrm{H} \alpha$ and $24 \mu \mathrm{m}$ peaks in M 51 and obtained $N=1.56 \pm 0.04$. Bigiel et al. (2008) studied the KS law for 18 nearby galaxies at $750 \mathrm{pc}$ resolution and derived $N=1.0 \pm 0.2$.

Recently, Verley et al. (2010) showed that the KS law breaks down at a smaller scale $(180 \mathrm{pc})$. This may indicate that the mechanism for triggering star formation operates at a scale 
Table 1

Parameters of NGC 4303

\begin{tabular}{|c|c|}
\hline Parameters & Value \\
\hline Name & NGC 4303/M 61 \\
\hline Hubble type ${ }^{a}$ & $\mathrm{SABbc}$ \\
\hline$V_{\mathrm{lsr}}^{\mathrm{b}}$ & $1556.5 \mathrm{~km} \mathrm{~s}^{-1}$ \\
\hline Distance $^{c}$ & $16.1 \mathrm{Mpc}$ \\
\hline Linear scale of $1^{\prime \prime}$ & $78.1 \mathrm{pc}$ \\
\hline \multicolumn{2}{|l|}{ Diameter $^{\mathrm{a}}$} \\
\hline Major & 6.5 \\
\hline Minor & 5.8 \\
\hline Position angle ${ }^{\mathrm{d}}$ & 312.2 \\
\hline Inclination $^{\mathrm{d}}$ & 27.8 \\
\hline AGN type & Sy $2 / \mathrm{H}$ II \\
\hline
\end{tabular}

Notes.

a de Vaucouleurs et al. (1991).

b Koda \& Sofue (2006).

${ }^{\text {c }}$ Ferrarese et al. (1996).

d This study.

smaller than $500 \mathrm{pc}$ and that star formation is triggered by the environment of GMCs at a scale $\leqslant 500 \mathrm{pc}$. We study the relation between the gas and star formation at a scale of $\sim 250 \mathrm{pc}$.

NGC 4303 is a nearby barred spiral galaxy with face-on geometry and is the ideal target for this study. It is a member of the Virgo Cluster $(D=16.1 \mathrm{Mpc}$; Ferrarese et al. 1996), whose bar and spiral arms are resolved spatially and kinematically with CARMA (Table 1). Even a glance at an $\mathrm{H} \alpha$ image is enough to recognize the striking difference in star formation activity between the bar and spiral arms in this galaxy (see Koopmann et al. 2001; Figure 5). The CO emission from NGC 4303 has been observed with various radio telescopes, i.e., the Owens Valley Radio Observatory (OVRO; Schinnerer et al. 2002), Nobeyama Millimeter Array (NMA; Koda \& Sofue 2006), Berkeley-Illinois-Maryland Association (BIMA; Helfer et al. 2003), and the NRO45 (Kuno et al. 2007). We observe NGC 4303 with NRO45 and CARMA and combine the data to obtain a high fidelity image. We focus on discussion of SFR and SFE as tracers of star formation activity at high spatial resolution within the galaxy.

The outline of this paper is as follows. In Section 2, we present our CO $(J=1-0)$ observations and data analysis. Results are described in Section 3. SFR and SFE in the bar and spiral arms of NGC 4303 are discussed in Section 4. The KS law is discussed in Section 5. A summary is given in Section 6.

\section{OBSERVATIONS}

We observed the ${ }^{12} \mathrm{CO}(J=1-0)$ line emission $\left(f_{\text {rest }}=\right.$ $115.2712 \mathrm{GHz}$ ) toward the barred spiral galaxy NGC 4303 with NRO45 and CARMA. The combination of NRO45 and CARMA interferometer data provides an unprecedented level of image fidelity at a high resolution. Interferometers have an intrinsic limitation: they cannot obtain the shortest spacing components in $u-v$ plane and thus are not sensitive to the most extended structures. The single-dish NRO45 covers the shortspacing data. The combination of the two telescopes is ideal for the study of extended nearby galaxies, enabling accurate measurements of surface brightnesses and gas surface densities. The observing parameters are listed in Table 2.

\subsection{NRO45}

The single-dish NRO45 observations were carried out in 2008 March at NMA. The 25 beam array, BEARS (Sunada et al. 2000), consists of double-sideband (DSB) superconductorinsulator-superconductor (SIS) receivers on a $5 \times 5$ grid with a separation of $\approx 41^{\prime \prime}$. 1 at $115 \mathrm{GHz}$. We used the digital autocorrelator for the NRO45 (Sorai et al. 2000) with a bandwidth of $512 \mathrm{MHz}\left(\sim 1340 \mathrm{~km} \mathrm{~s}^{-1}\right)$ and $500 \mathrm{kHz}$ resolution $\left(1.3 \mathrm{~km} \mathrm{~s}^{-1}\right.$ at $115 \mathrm{GHz})$.

The chopper wheel method was used for atmospheric and antenna ohmic loss corrections and to calibrate the antenna temperature in the double-sideband (DSB) $T_{\mathrm{A}}^{*}$ (DSB). A typical system temperature $T_{\text {sys }}$ was $300-400 \mathrm{~K}$ in the DSB during our observations. $T_{\mathrm{A}}^{*}(\mathrm{DSB})$ was converted to the single-sideband (SSB) temperature $T_{\mathrm{A}}^{*}$ (SSB) by applying scale factors (hereafter, we use $T_{\mathrm{A}}^{*}$ to refer to $T_{\mathrm{A}}^{*}(\mathrm{SSB})$ ). The scale factors were measured by comparing images of the Orion IRc2 region in the $\mathrm{CO}$ $(J=1-0)$ line obtained using BEARS and the singlebeam SSB receiver $\mathrm{S} 100$. The main beam efficiency of NRO45 was $\eta_{\mathrm{MB}}=0.30$ at $115 \mathrm{GHz}$. The main beam temperature is calculated as $T_{\mathrm{MB}}=T_{\mathrm{A}}^{*} / \eta_{\mathrm{MB}}$.

The telescope pointing was checked roughly every 30 minutes by observing the nearby $\mathrm{SiO}$ maser source R-Crv with another SIS receiver, S40 near $40 \mathrm{GHz}$. The standard deviation of the pointing offset between the optical axes of S40 and BEARS was 0 .'87, which is measured by the observatory. The pointing error was less than $6^{\prime \prime}$ during our observations. We mapped the $8^{\prime} \times$ $8^{\prime}$ region centered on NGC 4303 with the OTF-observing mode (Sawada et al. 2008), which corresponds to a $37 \mathrm{kpc} \times 37 \mathrm{kpc}$ area at the distance of NGC 4303. This area covers the optical extent of the galaxy. We alternated between continuous scans in R.A. and decl., as systematic noise appears dominantly along the direction perpendicular to the scan, which causes it to cancel out when the scans are combined. The 25 beams draw a regular $5^{\prime \prime}$ stripe in a single scan, which is finer than the Nyquist spacing of the $15^{\prime \prime}$ NRO45 beam-no spatial information is missed, which is critical in producing good $u-v$ coverage and in combining with CARMA data. We construct data cubes only for the $5^{\prime} \times$ $5^{\prime}$ area covered by all 25 beams (Figure 1 ).

We used the data reduction package Nobeyama OTF Software Tools for Analysis and Reduction (NOSTAR) developed at NRO (Sawada et al. 2008). After the DSB to SSB conversion, we subtracted spectral baselines with a first-order polynomial fit and flagged bad integrations. The R.A. and decl. scans provide data points roughly along a $5^{\prime \prime}$ lattice. We used a spheroidal function

Table 2

NRO45 and CARMA Observational Parameters

\begin{tabular}{lcc}
\hline \hline \multicolumn{1}{c}{ Parameters } & NRO45 & CARMA \\
\hline Date & 2008 March $9-12$ & 2007 October-November, 2008 June \\
Field center (J2000) & $12^{\mathrm{h}} 21^{\mathrm{m}} 54^{\mathrm{s}} .90,+4^{\mathrm{d}} 28^{\mathrm{m}} 25^{\mathrm{s}} .1$ & $12^{\mathrm{h}} 21^{\mathrm{m}} 54^{\mathrm{s}} .9,+4^{\mathrm{d}} 28^{\mathrm{m}} 25^{\mathrm{s}} .6$ \\
Observed frequency & $114.67641 \mathrm{GHz}$ & $114.67641 \mathrm{GHz}$ \\
Observed region & $8^{\prime} \times 8^{\prime}(37 \mathrm{kpc} \times 37 \mathrm{kpc})$ & $19 \mathrm{Points} \mathrm{mosaicing}$ \\
Bandwidth & $512 \mathrm{MHz}$ & $3 \times 62 \mathrm{MHz}$ \\
\hline
\end{tabular}




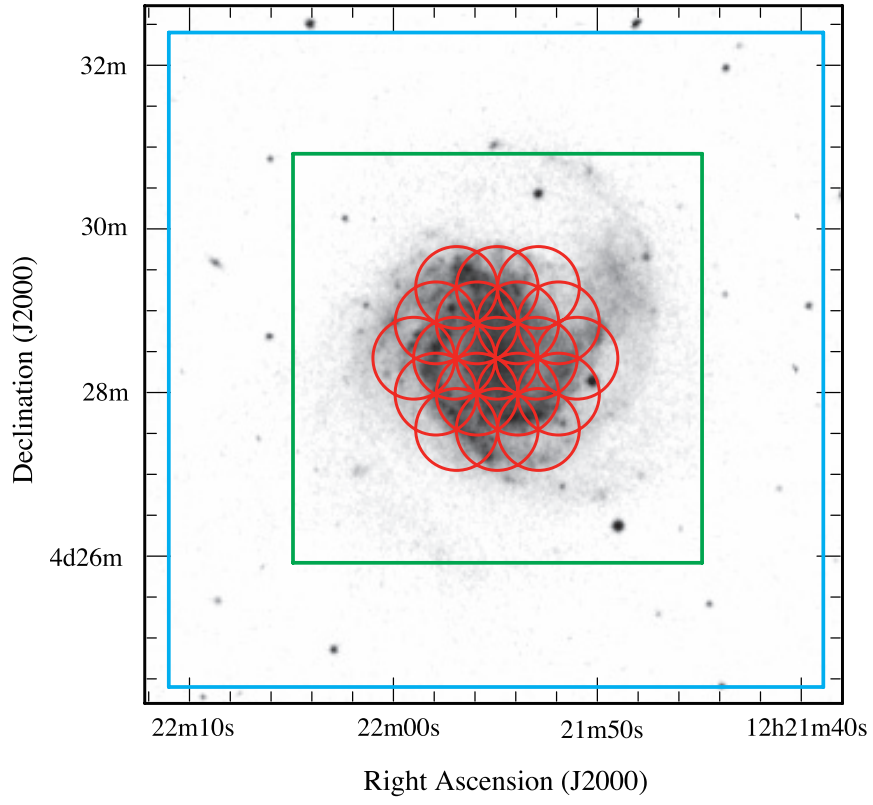

Figure 1. Optical image of NGC 4303 overlaid with regions observed using the NRO45 (blue outline) and CARMA (red circles). The 25 BEARS beams are spread evenly inside the region outlined in green, which we used for data reduction.

to regrid them onto a regular grid to produce an integrated data cube. The spheroidal function has low sidelobes in the Fourier domain and is advantageous in deconvolving the map to produce $u-v$ data. The convolution smears out the resolution of the map produced by the $15^{\prime \prime}$ NRO45 beam to $22^{\prime \prime} .1$. We produced separate data cubes for the scans taken in the R.A. and decl. directions and combine them using the basket-weave method in NOSTAR. Lower weights were applied to the data at small wave-numbers in the scan directions in the Fourier domain, which significantly reduces the systematic noise in the combined map.

The final data cube has a spatial resolution of 22 '. 1 and a pixel scale of 7".5, which is the half-size of $15^{\prime \prime}$ NRO45 beam. The rms noise is $75 \mathrm{mK}$ at the velocity resolution of $\Delta V=2.6 \mathrm{~km} \mathrm{~s}^{-1}$.

\subsection{CARMA}

We observed the central 2:3 region of NGC 4303 with CARMA in the C-configuration (2007 October and November) and in the D-configuration (2008 June). CARMA is a 15-element array, which consists of nine $6.1 \mathrm{~m}$ antennas (formerly BIMA) and six $10.4 \mathrm{~m}$ antennas (formerly OVRO). The angular resolution and baseline coverage of $\mathrm{C}$-configuration (D-configuration) are $2^{\prime \prime}\left(5^{\prime \prime}\right)$ and 26-370 $\mathrm{m}$ (11-148 m). The spatial resolution corresponding to the minimum baseline $(11 \mathrm{~m})$ is $49^{\prime \prime}$. We adopted a 19-point hexagonal mosaic pattern with $30^{\prime \prime}$ spacing (i.e., the Nyquist spacing of a $10.4 \mathrm{~m}$ primary beam; Figure 1). We used a $3 \mathrm{~mm}$ SIS receivers, and digital correlators with three bands, each of which has $62 \mathrm{MHz}$ of bandwidth in an SSB with $977 \mathrm{kHz}$ resolution $\left(2.5 \mathrm{~km} \mathrm{~s}^{-1}\right.$ at $\left.115 \mathrm{GHz}\right)$.

The bright quasar, $3 \mathrm{C} 273$, was observed for bandpass and gain calibrations. We observed it roughly every 20 minutes to calibrate for atmospheric and instrumental gain variations. We corrected the telescope pointing by observing 3C273 with the $3 \mathrm{~mm}$ receiver. We frequently monitored and corrected the pointing during tracks, using the optical pointing method developed by Corder (2008), which involves observing bright optical stars close to 3C273 every gain calibration cycle (around
20 minutes). The pointing offset vector between the radio and optical pointings was measured at the beginning of the observations.

We used the data reduction package MIRIAD (Sault et al. 1995) for calibration and data analysis. The rms noise level of the CARMA data is $43 \mathrm{mJy}^{\text {beam }}{ }^{-1}$ in a channel of $\Delta V=$ $2.6 \mathrm{~km} \mathrm{~s}^{-1}$. The synthesized beam size is 3 '. $1 \times 2$ '.5.

\subsection{Combining NRO45 and CARMA Data in $u-v$ Space}

We combined CARMA and NRO45 data in the Fourier domain as done in Koda et al. (2009) and Kurono et al. (2009). The NRO45 provided the short-spacing data which filled the central hole in the CARMA $u-v$ coverage. The combined image achieved higher angular resolution than the NRO45 map and recovered the flux missing in the CARMA map.

By utilizing MIRIAD $D^{5}$ and new routines that we developed, we combined NRO45 and CARMA data in the following way.

1. Deconvolution of single-dish map with single-dish beam. The point-spread function (PSF) of the NRO45 map is a convolution of the NRO45 beam pattern and the spheroidal function that we applied to regrid the data points and create the final data cube. The map should be deconvolved by the PSF before being Fourier transformed to the $u-v$ plane. The NRO45 beam size is Gaussian with an effective beam size of 22 ". 1 .

2. Multiplying dummy 2' Gaussian primary beam. This step produced the same data structure for the single-dish data as is used by the interferometer data in order for the files to be read into MIRIAD. We used a 19 point hexagonal mosaic technique in our CARMA observations, so the NRO45 map was divided into 19 smaller maps corresponding to CARMA's mosaic points. The pseudo-primary beams were multiplied by each of the 19 maps to mimic CARMA observations. CARMA has three types of primary beam pattern with an FWHM of $64^{\prime \prime}$ for $10.4 \mathrm{~m}-10.4 \mathrm{~m}, 85^{\prime \prime}$ for $6.1 \mathrm{~m}-10.1 \mathrm{~m}$, and $115^{\prime \prime}$ for $6.1 \mathrm{~m}-6.1 \mathrm{~m}$ at $115 \mathrm{GHz}$. To mimic this behavior, we applied a Gaussian primary beam with an FWHM of $2^{\prime}$ to the deconvolved NRO45 map at each of the 19 pointings and produced 19 maps. The Fourier transformations of these maps are the $u-v$ data we need (see step 4).

3. Determining weightings. The relative weighting of NRO45 and CARMA data changes the synthesized beam pattern. By changing the weighting, we can control the sidelobes of the synthesized beam and the noise level. This is similar to the conventional weighting schemes (e.g., natural and uniform weighting or robust parameter). We decided to use the weighting that minimized the sidelobes.

4. Constructing visibility data from NRO45 data. All of the visibility data of NRO45 are built after applying the relative weight balance between the visibility data of NRO45 and CARMA (Kurono et al. 2009). We made 57 visibility data sets, i.e., for 19 pointings $\times 3$ primary beams. All $57 \mathrm{NRO} 45$ visibility data sets are concatenated to produce the final $\mathrm{NRO} 45 u-v$ data.

5. Imaging. We obtain the combined data cube by adding together the visibility data sets from NRO45 and CARMA, and by Fourier transforming the result, we created a combined image. The total flux of the combined image is similar to that of the NRO45 image. The rms noise of the

\footnotetext{
5 See http://bima.astro.umd.edu/miriad/
} 

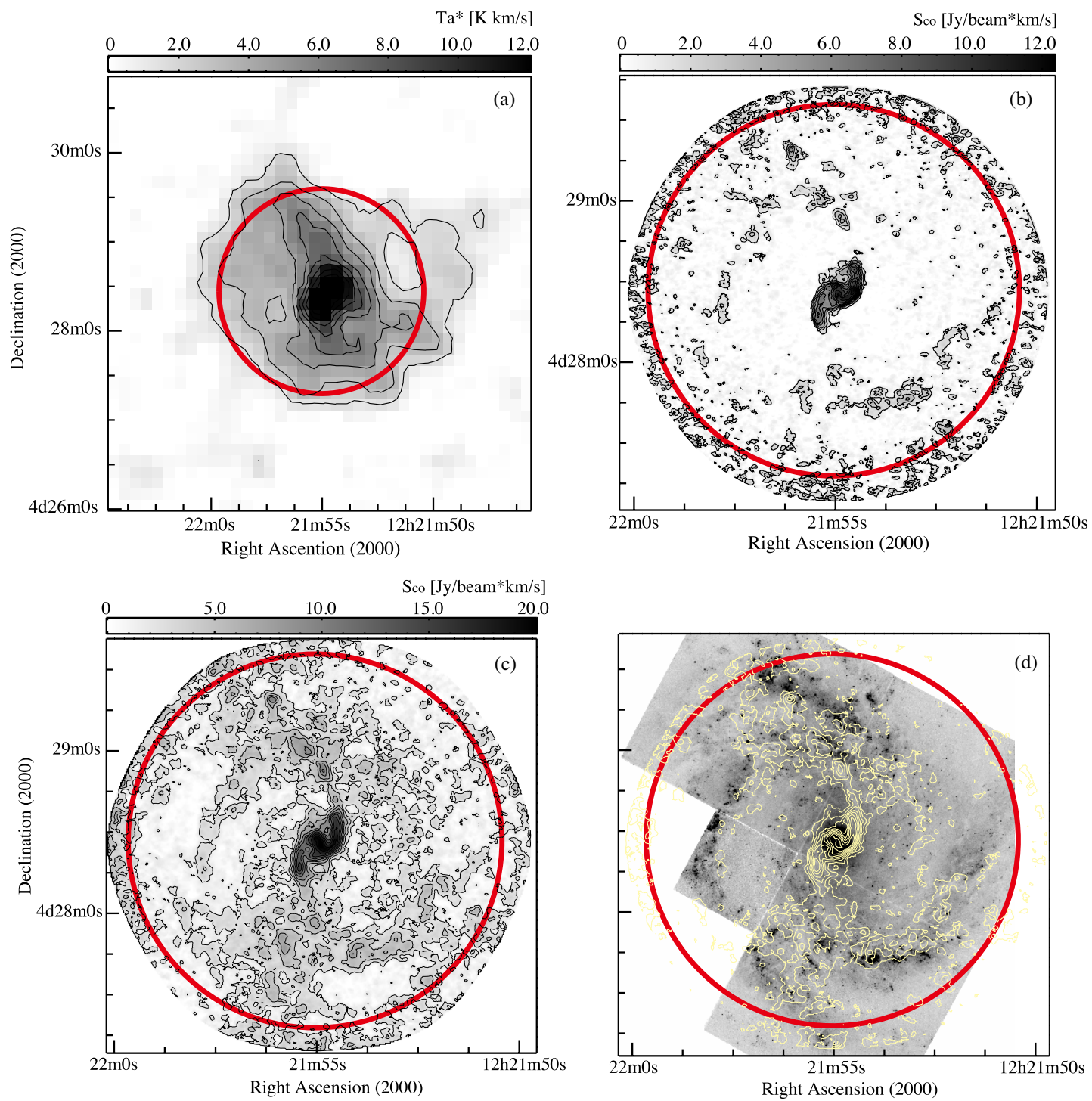

Figure 2. Integrated intensity map for (a) NRO45, (b) CARMA, (c) NRO45 + CARMA (combined data), and (d) combined data overlaid on HST F450 image. The contours trace $10 \%$ intervals from $10 \%$ to $90 \%$ of the peak. $1 \sigma$ noise levels are $1.5 \mathrm{~K} \mathrm{~km} \mathrm{~s}^{-1}$ (NRO45), $810 \mathrm{mJy} \mathrm{beam}^{-1} \mathrm{~km} \mathrm{~s}^{-1}$ (CARMA), and $710 \mathrm{mJy} \mathrm{beam}^{-1} \mathrm{~km} \mathrm{~s}^{-1}$ (combined data). The peak intensities of the NRO45, CARMA, and NRO45 + CARMA images are $15.7 \mathrm{~K} \mathrm{~km} \mathrm{~s}^{-1}, 13.0 \mathrm{Jy}$ beam ${ }^{-1} \mathrm{~km} \mathrm{~s}^{-1}$, and $20.8 \mathrm{Jy}_{\text {beam }}{ }^{-1} \mathrm{~km} \mathrm{~s}^{-1}$, respectively. The NRO45 + CARMA image achieves a high resolution as well as recovering extended emission components. The CO emission overlaps with dust lanes, which are seen as extinction in the HST image. Red circles are the central 2!3 region observed with CARMA.

Table 3

NRO45, CARMA, and Combined Data Results

\begin{tabular}{lccc}
\hline \hline \multicolumn{1}{c}{ Parameters } & NRO45 & CARMA & Combined Data \\
\hline Velocity resolution $\Delta V$ & $2.6 \mathrm{~km} \mathrm{~s}^{-1}$ & $2.6 \mathrm{~km} \mathrm{~s}^{-1}$ & $2.6 \mathrm{~km} \mathrm{~s}^{-1}$ \\
The rms noise in a channel & $73 \mathrm{mK}^{-1}$ & $43 \mathrm{mJy} \mathrm{beam}^{-1}$ & $34 \mathrm{mJy} \mathrm{beam}^{-1}$ \\
The rms noise in an intensity map & $1.5 \mathrm{~K} \mathrm{~km} \mathrm{~s}^{-1}$ & $810 \mathrm{mJy} \mathrm{beam}^{-1} \mathrm{~km} \mathrm{~s}^{-1}$ & $710 \mathrm{mJy} \mathrm{beam}^{-1} \mathrm{~km} \mathrm{~s}^{-1}$ \\
Beam/Synthesized beam size & $22^{\prime \prime} 1$ & $3 . \prime 1 \times 2.5$ & $3.2 \times 3^{\prime \prime} .2$ \\
Total flux & $2.8 \times 10^{3} \mathrm{Jy} \mathrm{km} \mathrm{s}^{-1}$ & $9.8 \times 10^{2} \mathrm{Jy} \mathrm{km} \mathrm{s}^{-1}$ & $2.9 \times 10^{3} \mathrm{Jy} \mathrm{km} \mathrm{s}^{-1}$ \\
\hline
\end{tabular}

combined cube is $34 \mathrm{mJy}_{\text {beam }}{ }^{-1}$ with $\Delta V=2.6 \mathrm{~km} \mathrm{~s}^{-1}$. The synthesized beam is 3 .'2 2 3".2.

Bright $\mathrm{CO}$ emission is detected throughout the disk of NGC 4303, even in the inter-arm region in NRO45 map (Figure 2(a)). On the other hand, the CARMA map shows emission only from a compact component in the nuclear region and the spiral arms (Figure 2(b)). The combined data map clearly shows strong $\mathrm{CO}$ emission in the nucleus, the bar, and the disk region along the two arms (Figure 2(c)). A comparison of the total flux in the NRO45, CARMA, and combined images clearly shows that the missing flux has been completely recovered (Table 3).

\subsection{Archival data}

We obtained archival $\mathrm{H} \alpha$ (Knapen et al. 2004) and $K$ band (Möllenhoff \& Heidt 2001) images from the NASA Extragalactic Database $\left(\mathrm{NED}^{6}\right)$ and a $24 \mu \mathrm{m}$ image (P.I.: Kotak)

\footnotetext{
6 See http://nedwww.ipac.caltech.edu/.
} 


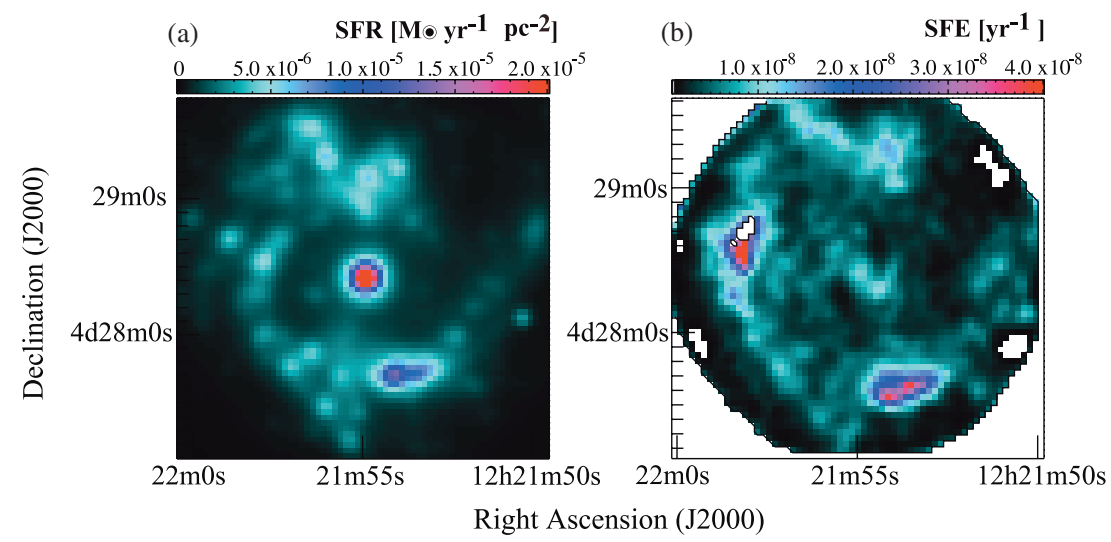

Figure 3. SFR map (left) and SFE map (right) of NGC 4303. SFR is calculated from the H $\alpha$ and Spitzer $24 \mu \mathrm{m}$ images so that the effect of extinction is small.

from the Spitzer Space Telescope data archive using the Leopard software. The retrieved $24 \mu \mathrm{m}$ image suffered from residual sky background emission, which we subtracted using the Image Reduction and Analysis Facility (IRAF ${ }^{7}$ ) package.

UV photons from recently formed, young, massive stars primarily ionize the surrounding gas (H II regions) or heat dust grains (thermal radiation). Thus, the combination of $\mathrm{H} \alpha$ and $24 \mu \mathrm{m}$ dust continuum emissions traces the SFR fairly accurately (Calzetti et al. 2007; Kennicutt et al. 2007). The SFR is calculated using the formulae from Kennicutt et al. (2007) and Calzetti et al. (2007), namely

$$
\begin{aligned}
\operatorname{SFR}\left(M_{\odot} \mathrm{yr}^{-1}\right)= & 7.9 \times 10^{-42} L(\mathrm{H} \alpha)_{\text {corr }}\left(\mathrm{erg} \mathrm{s}^{-1}\right) \\
= & 7.9 \times 10^{-42}\left(L(\mathrm{H} \alpha)_{\text {obs }}\right. \\
& +(0.031 \pm 0.006) L(24 \mu \mathrm{m}))\left(\mathrm{erg} \mathrm{s}^{-1}\right)
\end{aligned}
$$

where $L(\mathrm{H} \alpha)$ and $L(24 \mu \mathrm{m})$ are the $\mathrm{H} \alpha$ and $24 \mu \mathrm{m}$ luminosities. From the $\mathrm{H} \alpha$ and $24 \mu \mathrm{m}$ images, we found that $\mathrm{H} \alpha$ emission defined the sites of star-forming regions very well, although it does not quantitatively trace the SFR. Since the $\mathrm{H} \alpha$ image has a much higher resolution (1".5) than the $24 \mu \mathrm{m}$ image, we use the $\mathrm{H} \alpha$ image in discussing the offsets between star-forming regions and parental gas qualitatively. For quantitative measurements, we convolve the $\mathrm{H} \alpha$ data and smooth the $\mathrm{H} \alpha$ and $\mathrm{CO}$ images to match the spatial resolution of the $24 \mu \mathrm{m}$ image $\left(6^{\prime \prime} \sim 500 \mathrm{pc}\right)$. We clip the SFR and CO data at the $2 \sigma \mathrm{rms}$ noise level. The SFR map is shown in Figure 3 (left).

\section{RESULTS}

\subsection{Molecular Gas Distribution}

Molecular gas distributions in barred spiral galaxies have been studied by many authors, from high resolution observations of central bars (e.g., Kenney et al. 1993; Ishizuki 1999; Sakamoto et al. 1999a, 1999b) to lower resolution observations of larger disks (Sheth et al. 2000). The gas distribution in NGC 4303 is typical among barred spiral galaxies; it shows two narrow ridges (namely, offset ridges) at the leading side of the bar, and spiral arms in the outer part, and coincides with dust lanes in optical images. Figure 2(d) shows an overlay of $\mathrm{CO}$ contours on an archival HST F450 band image (P.I.: Smartt).

\footnotetext{
7 IRAF is distributed by the National Optical Astronomy Observatory, which is operated by the Association of Universities for Research in Astronomy, Inc., under cooperative agreement with the National Science Foundation.
}

The $\mathrm{CO}$ emission is concentrated in the central $40^{\prime \prime}$ $(\sim 3.2 \mathrm{kpc})$, which includes the offset ridges and circumnuclear disk. In particular, the circumnuclear disk shows a circular structure $(r=4.4 \sim 350 \mathrm{pc})$, extending from $1541.3 \mathrm{~km} \mathrm{~s}^{-1}$ southeast of the nucleus to $1611.5 \mathrm{~km} \mathrm{~s}^{-1}$ to the northwest in the velocity channel maps (Figures 4 and 5). This structure is called a ring or part of the offset ridges (i.e., inner spiral arms; Schinnerer et al. 2002; Koda \& Sofue 2006). The circumnuclear disk hosts a spiral arm in the UV continuum image (Colina et al. 1997), and the CO ring/spiral arm structure is offset from the UV spiral arm.

Low-level CO emission is also present in the space outside of the offset ridges (namely, inter-offset ridge regions). Strong concentrations of gas exist at the outer ends of the offset ridges (Figure 2(c)). Molecular spiral arms extend from those end points toward the outer regions. The map resolves the widths of the offset ridges of the bar and the outer spiral arms at high resolution. The surface densities of the gas in the offset ridges and outer spiral arms are not similar at the high resolution, differing by factor of $\sim 2$.

We derive the inclination and position angle of the galaxy using the task GAL in the AIPS data reduction package. The results are shown in Table 1. We use these values for the rest of our analysis.

\subsection{Molecular Gas Mass and Surface Density}

We estimate the gas mass from the CO-integrated intensity $S_{\mathrm{CO}}$ (see Figure 2(c)), using the Galactic $I(\mathrm{CO})$-to- $N\left(\mathrm{H}_{2}\right)$ conversion factor $X_{\mathrm{CO}}$. We adopt the standard value $X_{\mathrm{CO}}=2.0 \times$ $10^{20} \mathrm{~cm}^{-2}\left(\mathrm{~K} \mathrm{~km} \mathrm{~s}^{-1}\right)^{-1}$ (Strong 1987). The gas mass is then

$$
\begin{aligned}
M_{\mathrm{H}_{2}}= & 1.0 \times 10^{4}\left(\frac{S_{\mathrm{CO}}}{\mathrm{Jy} \mathrm{km} \mathrm{s}^{-1}}\right)\left(\frac{D}{\mathrm{Mpc}}\right)^{2} \\
& \times\left[\frac{X_{\mathrm{CO}}}{3.0 \times 10^{20} \mathrm{~cm}^{-2}\left(\mathrm{~K} \mathrm{~km} \mathrm{~s}^{-1}\right)^{-1}}\right] M_{\odot},
\end{aligned}
$$

where $D$ is the distance to the galaxy, which assumed to be 16.1 Mpc (Ferrarese et al. 1996). The total gas mass of the galaxy is calculated from the NRO45 map to be $5.3 \times 10^{9} M_{\odot}$. The CARMA mosaic covered the circular area with a radius of $80^{\prime \prime}$. The enclosed mass is $3.4 \times 10^{9} M_{\odot}$. The average gas surface density in the central $160^{\prime \prime}$ of the molecular disk is $36 M_{\odot} \mathrm{pc}^{-2}$. 


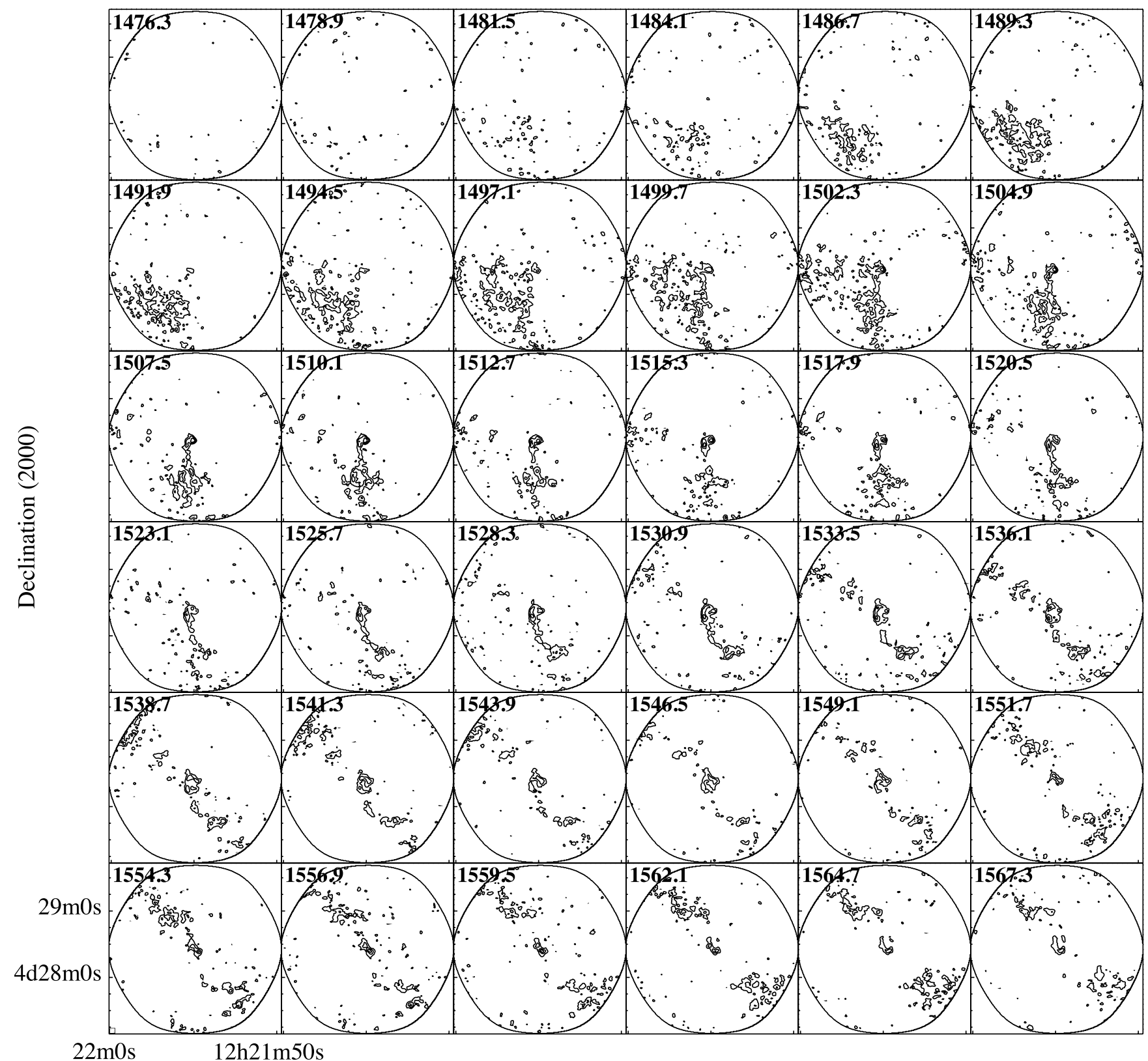

Right Ascension (2000)

Figure 4. Channel maps of the combined data from $1475.0 \mathrm{~km} \mathrm{~s}^{-1}$ to $1566.0 \mathrm{~km} \mathrm{~s}^{-1}$. The contours are $3 \sigma$, $6 \sigma$, and $9 \sigma$, where the $1 \sigma$ noise level is $34 \mathrm{mJy}$ beam ${ }^{-1}$.

\section{MOLECULAR GAS AND STAR FORMATION}

\subsection{Spatially Resolved Sites of Star Formation}

Both $\mathrm{CO}$ and $\mathrm{H} \alpha$ emission appear clearly along stellar spiral arms, and the $\mathrm{H} \alpha$ arms are offset toward the leading side of the $\mathrm{CO}$ arms. Similar offsets are evident between $\mathrm{CO}$ and $24 \mu \mathrm{m}$ emission. The $24 \mu \mathrm{m}$ emission is at the leading side of the CO emission. These offsets are due to the delay of star formation from the accumulation of gas in the spiral arms (Egusa et al. 2004); approximately, an offset would be $\left(\Omega-\Omega_{\mathrm{p}}\right){ }_{\mathrm{SF}}$, where $\Omega$ and $\Omega_{\mathrm{p}}$ are the angular speed of the gas and pattern speed of spiral arms, respectively, and $t_{\mathrm{SF}}$ is the timescale of star formation. The sites of star formation are resolved in our observations. The analysis of local SFR and efficiency should take this into account, since the parental gas and star-forming regions would not be correlated at the high spatial resolution.
This means that the KS law breaks down at this resolution. We need to smooth the data before characterizing the spatial variation of the SFE in Section 4.2.

Figures 6(a) and (b) show a close-up of the offset between $\mathrm{CO}$ (black contours) and $\mathrm{H} \alpha$ emissions (gray scale). In the upper panels, the arrows indicate the direction of gas flow, assuming pure azimuthal rotation and a time lag between star formation and gas accumulation in spiral arms. Figure 6(c) shows the phase diagrams of $\mathrm{CO}$ (contours) and $\mathrm{H} \alpha$ (gray scale). Again, the $\mathrm{H} \alpha$ emission is found predominantly at the leading side of the CO spiral arms over the entire range of radii (over $40^{\prime \prime}$, $\sim 3.1 \mathrm{kpc}$ ).

The offsets between the $\mathrm{CO}$ and $\mathrm{H} \alpha$ arms range from 0 to $1 \mathrm{kpc}$, with an average of $\sim 500 \mathrm{pc}$ at radius, $r \geqslant 40^{\prime \prime}(\sim 3.1 \mathrm{kpc}$; i.e., the area we define as outer spiral arms in Section 4.4). Thus, we will smooth the data to the spatial resolution of $\sim 500 \mathrm{pc}$ for 


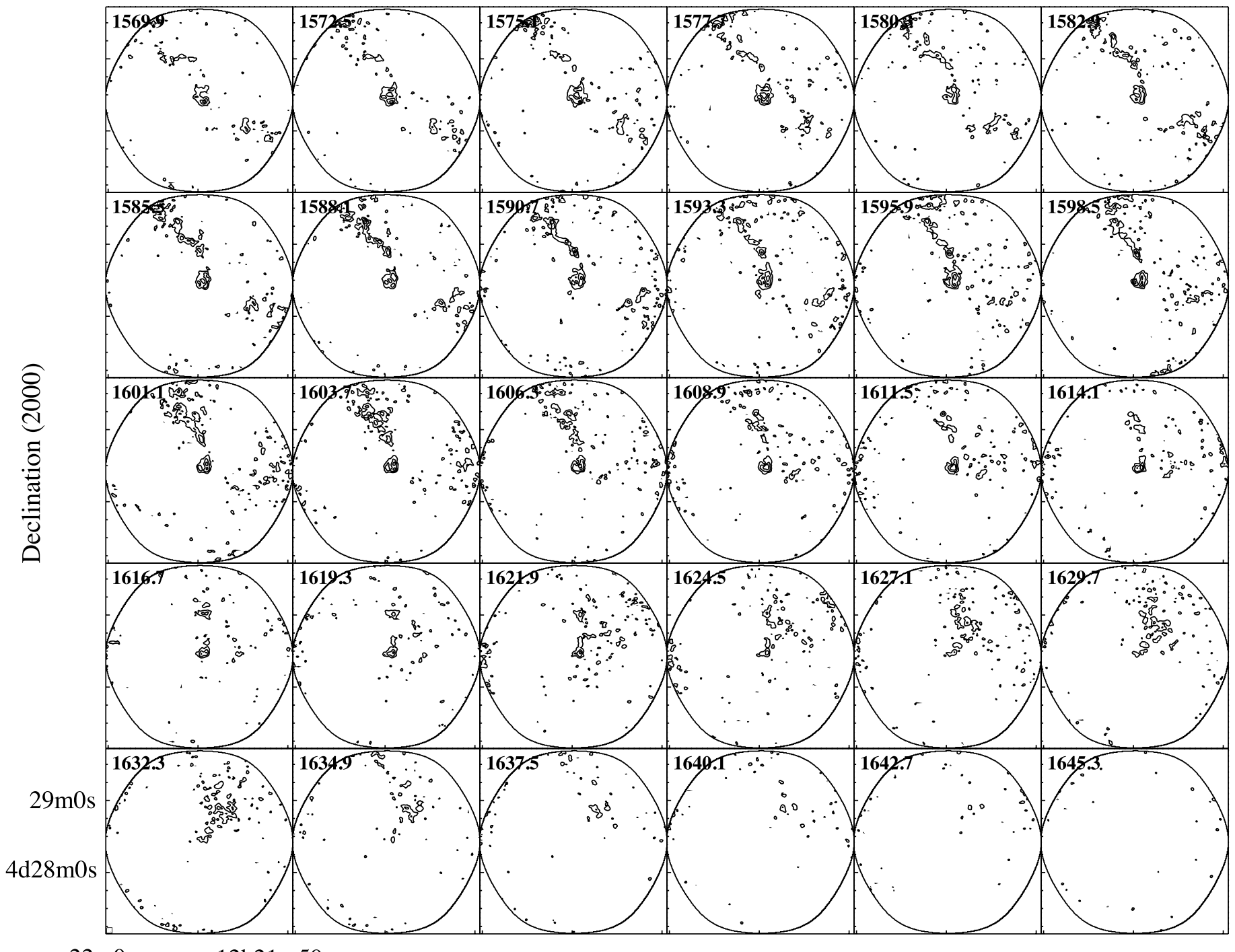

$22 \mathrm{~m} 0 \mathrm{~s} \quad 12 \mathrm{~h} 21 \mathrm{~m} 50 \mathrm{~s}$

Right Ascension (2000)

Figure 5. Channel maps from $1568.6 \mathrm{~km} \mathrm{~s}^{-1}$ to $1644.0 \mathrm{~km} \mathrm{~s}^{-1}$.

later discussions; it also especially affects the analysis of SFE. We will discuss this further in Section 4.4.

Egusa et al. (2004) introduced a new method to estimate the timescale of star formation using these offsets, measuring the offset/time lag between gas accumulation and star formation. Tamburro et al. (2008) applied this method to $\mathrm{HI}$ and $24 \mu \mathrm{m}$ data. Their offsets $\leqslant 250 \mathrm{pc}$ (calculated from their paper) are translated to the timescale of $\sim 1$ myr. On the other hand, Egusa et al. (2009) used CO and $\mathrm{H} \alpha$ data for NGC 4303 and derived the offsets of 700-1000 pc and the timescale of $\sim 10$ myr, an order of magnitude longer than the Tamburro et al. (2008) results. Our offsets are closer to those in Egusa et al. (2009). To understand this discrepancy, we compared $24 \mu \mathrm{m}$ and $\mathrm{H} \alpha$ images and found no significant offsets between the two. Thus, $\mathrm{H} \alpha$ traces the locations of star-forming regions as well as $24 \mu \mathrm{m}$. The discrepancy may come from the difference of $\mathrm{CO}$ and $\mathrm{H}$ I. If the $\mathrm{H}$ i emission traces the gas dissociated by star formation, and not the natal gas for star formation, it explains the small offsets between $\mathrm{HI}_{\mathrm{I}}$ and $24 \mu \mathrm{m}$ emissions. Our simple analysis with $\mathrm{CO}$ and $\mathrm{H} \alpha / 24 \mu \mathrm{m}$ favors the longer star formation timescale of Egusa et al. (2009) in the case of NGC 4303. These results should be confirmed with a larger sample of galaxies.

\subsection{Star Formation Rate and Efficiency}

We compare the SFR and SFE between the bar and outer spiral arms, as well as within other components (i.e., circumnuclear disk, inter-arm regions), at the spatial resolution of $\sim 500 \mathrm{pc}$. The SFE is the ratio of the SFR to the gas mass within an aperture, namely

$$
\operatorname{SFE}\left(\mathrm{yr}^{-1}\right)=\frac{\operatorname{SFR}\left(M_{\odot} \mathrm{yr}^{-1}\right)}{M_{\mathrm{H}_{2}}\left(M_{\odot}\right)},
$$

where $M_{\mathrm{H}_{2}}$ is the molecular hydrogen gas mass. We calculate the SFR and gas mass within an aperture and use the averaged parameters to derive the SFE. We smooth our $\mathrm{CO}$ and $\mathrm{H} \alpha$ images to the same resolution as the $24 \mu \mathrm{m}$ image ( $6^{\prime \prime}$ resolution) in order to correlate the parental gas and star-forming regions. This resolution corresponds to a linear size of $\sim 500 \mathrm{pc}$, similar to the linear resolution at which Kennicutt et al. (2007) find the correlation between gas surface density and associated SFR.

A SFR map is shown in Figure 3(a). High SFRs are apparent in intense star-forming regions which are sparsely distributed along the spiral arms, as well as in the circumnuclear disk. However, SFRs are lower in the bar, even though the gas surface densities along the bar offset ridges are as high as those in 

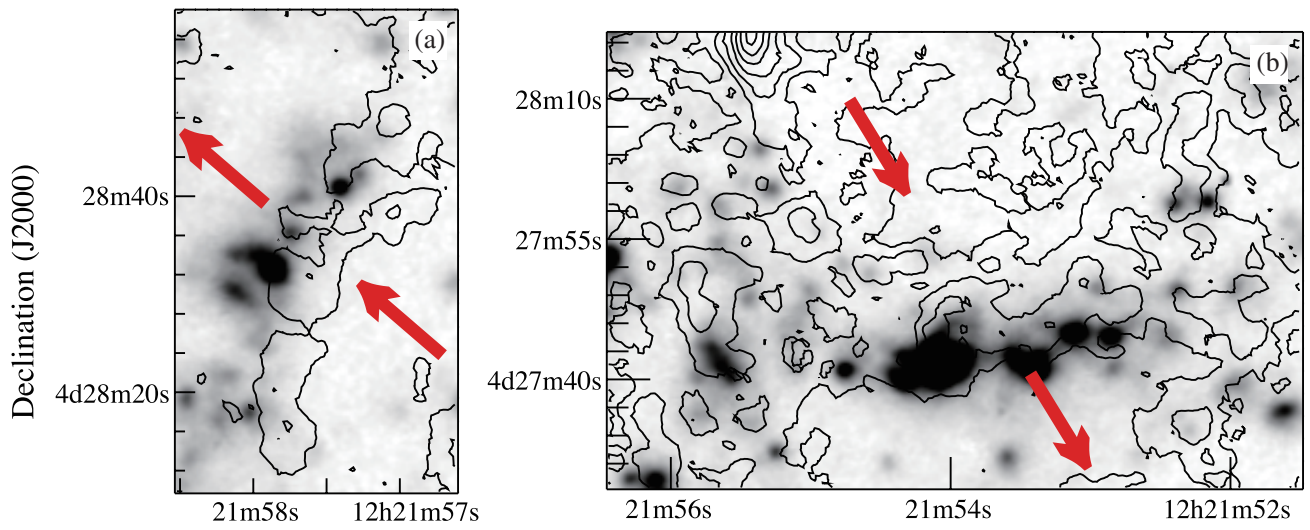

Right Ascension (J2000)

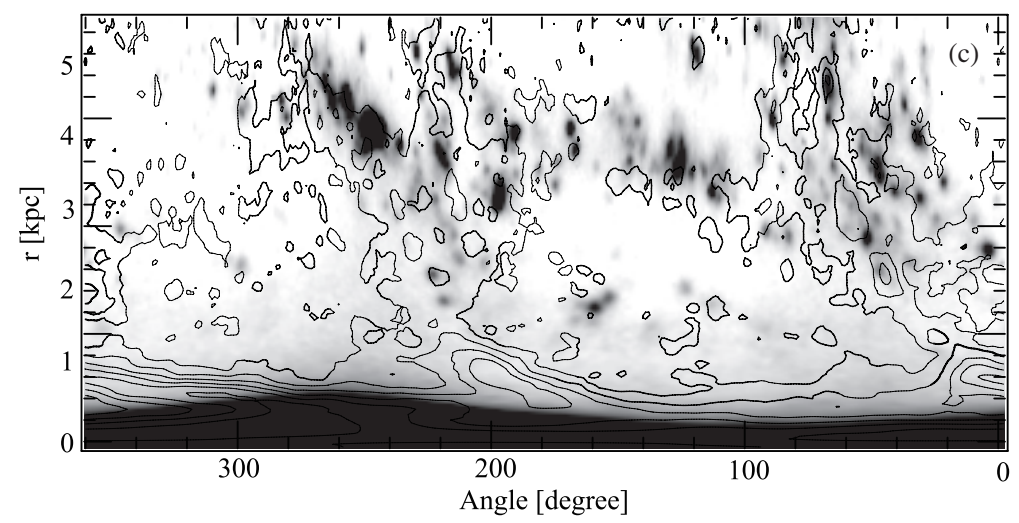

Figure 6. (a) and (b): Distributions of CO (black contours) and $\mathrm{H} \alpha$ (gray scale) emission in two regions of NGC 4303 are shown. The direction of gas flow is determined assuming a circular galactic rotation and that the young massive stars (traced by H $\alpha$ ) flow downstream from the molecular spiral arms where they were formed. (c) The phase diagram of $\mathrm{CO}$ (contours) and $\mathrm{H} \alpha$ (gray scale).

the spiral arms. The low SFR in the bar has been suggested qualitatively by previous studies (most systematically by Sheth et al. 2002), and we discuss this quantitatively in the following sections.

Figure 3(b) shows a map of the SFE. Low SFEs are seen in the bar (especially around the offset ridges) and in the inter-arm regions. Exceptionally high SFEs appear at the leading side of the $\mathrm{CO}$ arms. This indicates that the offsets between $\mathrm{H} \alpha$ and $\mathrm{CO}$ emission are not fully smoothed out even at the resolution of $\sim 500 \mathrm{pc}$ (see Section 3.2), even though the parental gas shows a fair correlation with SFR (Kennicutt et al. 2007).

\subsection{Azimuthally Averaged Star Formation Efficiency}

We first compare the gas surface density $\left(\Sigma_{\mathrm{H}_{2}}\right)$, area-averaged SFR $\left(\Sigma_{\text {SFR }}\right)$, and SFE between the bar and spiral arms on the basis of azimuthal averages (Figure 7). The average values are derived in $1 \mathrm{kpc}\left(12^{\prime \prime} .8\right)$ width annuli, which is enough to average out the spatial offsets (see Section 3.2). The $1 \mathrm{kpc}$ resolution can still separate the bar, spiral arms, and nucleus without significant contamination from each other. Figure 8(a) shows the definitions of nucleus, bar, and outer spiral arms. The nucleus might include a portion of the bar region; however, we are mainly interested in the differences between the bar and spiral arms, so it does not affect our analysis. We will comment on the properties of the circumnuclear star formation in Section 4.4.

Figure 7 shows the radial distributions of the area-averaged SFR $\Sigma_{\text {SFR }}$ (a), gas surface density $\Sigma_{\mathrm{H}_{2}}$ (b), and SFE (c). The definitions of the nucleus, bar, and spiral arms are indicated with arrows in Figure 7(a). SFE drops abruptly from the circumnuclear disk to the bar and increases toward the spiral arms (Figure 7(c)). Quantitatively, the average SFE is about twice as high in the spiral arms as in the bar.

\subsection{Locally Averaged Star Formation Efficiency}

The SFE (Equation (3)) is a correlation between star-forming regions and their parental gas. We have resolved the scale where the star-forming regions and gas are spatially separated; therefore, we have to smooth the data to derive an SFE with physical importance. Kennicutt et al. (2007) found a good correlation between the surface densities of SFR and gas mass at the $500 \mathrm{pc}$ scale. This is similar to the typical separation of $\mathrm{H} \alpha$ and CO emission that we see in NGC 4303 (Section 4.1). We smooth $\mathrm{H} \alpha$ and our CO images to the scale of the Spitzer $24 \mu \mathrm{m}$ image (6"; $\sim 500 \mathrm{pc})$ and compare local SFR and SFE between the bar and spiral arms at this scale.

Four regions (i.e., bar, spiral arms, inter-arms regions, and circumnuclear region) are defined as in Figure 8(b). The circumnuclear region is a $6^{\prime \prime}$ diameter circle $(\sim 500 \mathrm{pc})$, based on a near-infrared (NIR) image of Pérez-Ramírez et al. (2000). We define the bar as an ellipse with the deprojected major and minor axis diameters of $40^{\prime \prime} \times 26^{\prime \prime}(3.1 \times 2.0 \mathrm{kpc})$, based on a $K$-band image. This definition yields an ellipticity similar to the one derived by Laine et al. (2002) based on a near-infrared $\mathrm{H}$-band image and a major axis diameter consistent with the determination of Schinnerer et al. (2002). The spiral arms and inter-arm regions are separated by eye using the $K$-band image. Figure 8(b) shows our definitions overlaid on the $K$-band image. 

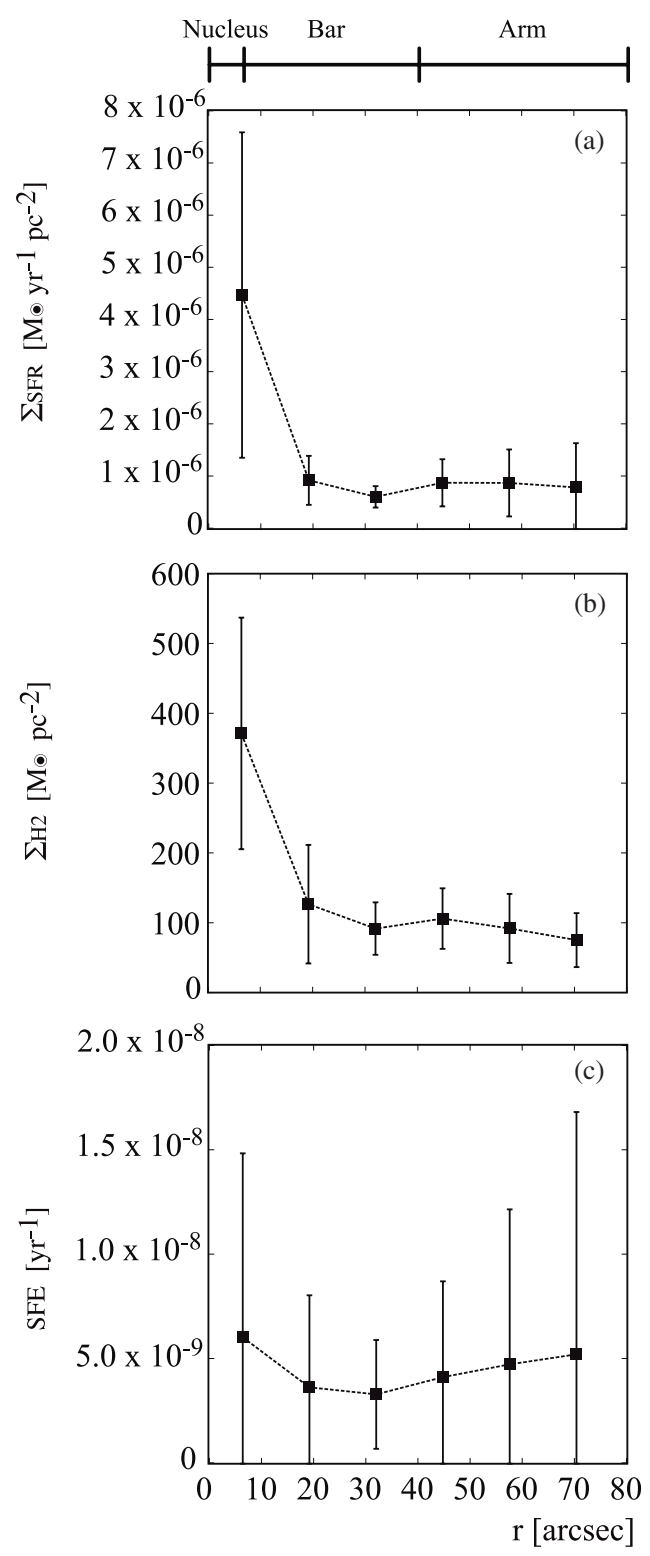

Figure 7. Radial distributions of (a) $\Sigma_{\mathrm{SFR}}$, (b) $\Sigma_{\mathrm{H}_{2}}$, and (c) SFE. The error bars are one rms.
Table 4

Average of SFR and SFE over the Entire Disk Regions, Bar, and Spiral Arms

\begin{tabular}{lccc}
\hline \hline & The Entire Disk & The Bar & The Spiral Arms \\
\hline SFR $\left(M_{\odot} \mathrm{yr}^{-1}\right)$ & $8.3 \times 10^{-2}$ & $7.6 \times 10^{-2}$ & $9.8 \times 10^{-2}$ \\
SFE $\left(\mathrm{yr}^{-1}\right)$ & $5.7 \times 10^{-9}$ & $4.2 \times 10^{-9}$ & $7.4 \times 10^{-9}$
\end{tabular}

Figure 9 shows the histograms of $\Sigma_{\mathrm{H}_{2}}$ (left), $\Sigma_{\mathrm{SFR}}$ (center), and SFE (right) for the spiral arms and bar (top and bottom). High gas surface density regions are present only in the bar, but not in the spiral arms. However, high SFR and SFE regions appear only in the spiral arms and are absent in the bar. Therefore, high SFR and SFE are evident in the spiral arms in the local average, as well as in the azimuthal average.

We calculate the excesses of SFR and SFE in each region with respect to the averages over the entire disk excluding the nucleus (Table 4). The SFR and SFE in the bar are 10\% and 30\% smaller than the disk averages, respectively. Star formation activity in the bar is low compared to the entire disk. On the contrary, the SFR and SFE in the spiral arms are about $10 \%$ and $40 \%$ higher than those over the disk, respectively. Active star formation preferentially occurs in the spiral arms. SFR and SFE are about $30 \%$ and twice as high in the spiral arms as in the bar. The results confirm quantitatively the notion that star formation in bar is less than in spiral arms.

The effect of the spatial offsets between $\mathrm{H} \alpha$ and $\mathrm{CO}$ emissions is significantly reduced by the smoothing to estimate local SFE ( $\sim 500 \mathrm{pc}$ resolution). However, it still shows some residual effects in SFEs, since the average of this offset is also $\sim 500 \mathrm{pc}$. If the offset is below $500 \mathrm{pc}$, parent GMCs and star-forming regions exist within our resolution for SFE discussions. If the offset is above $500 \mathrm{pc}$, we cannot recognize both parent GMCs and star-forming regions within the resolution. SFEs obtained in such a region will not fairly represent the local SFE. This produces SFEs above $10^{-7.8} \mathrm{yr}^{-1}$ are the artifacts (Figure 3).

\subsection{Circumnuclear Star Formation}

The SFR and SFE are the highest in the nuclear region. The azimuthal averages of $\Sigma_{\text {SFR }}$ and SFE (Figures 7(a) and (c)) clearly show this tendency, being about 5 times and twice higher than the disk averages, respectively. The locally averaged SFE in the nucleus is also high. The locally averaged SFR and SFE
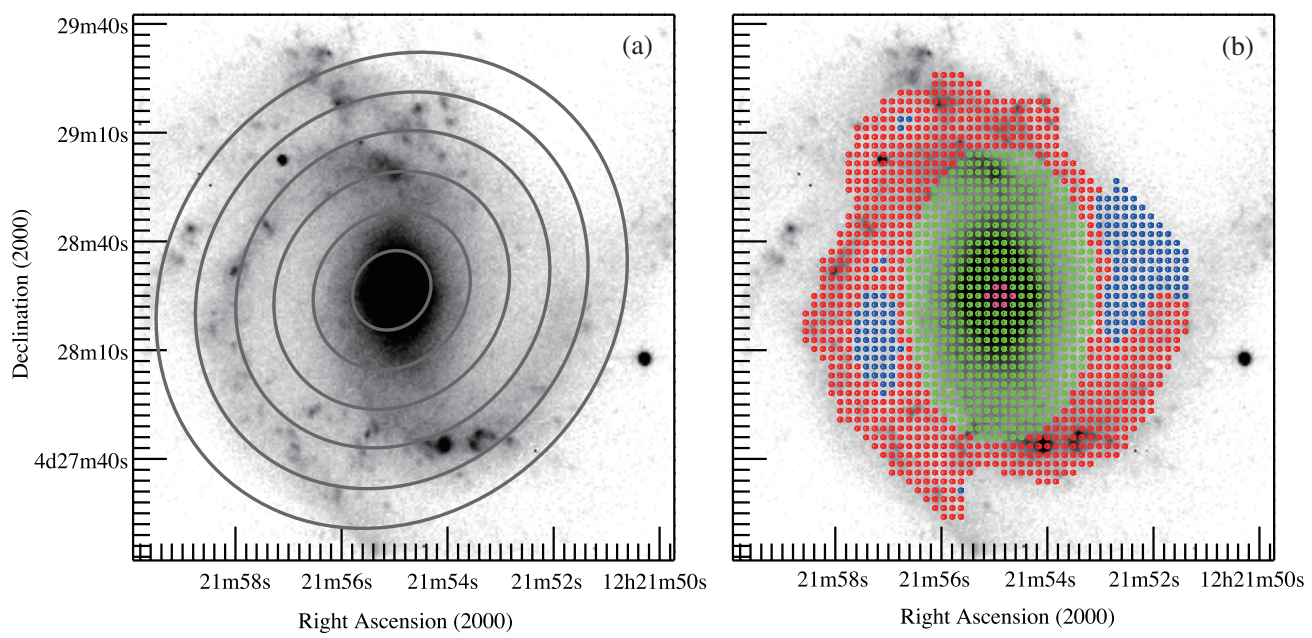

Figure 8. Definitions of the nucleus, bar, and spiral arm (outer disk) regions. Left: azimuthal definitions. Each annulus has 1 kpc width. Right: local definitions with $3^{\prime \prime}$ grid. The nucleus, bar, arm, and inter-arm are represented by pink, green, red, and blue, respectively. 

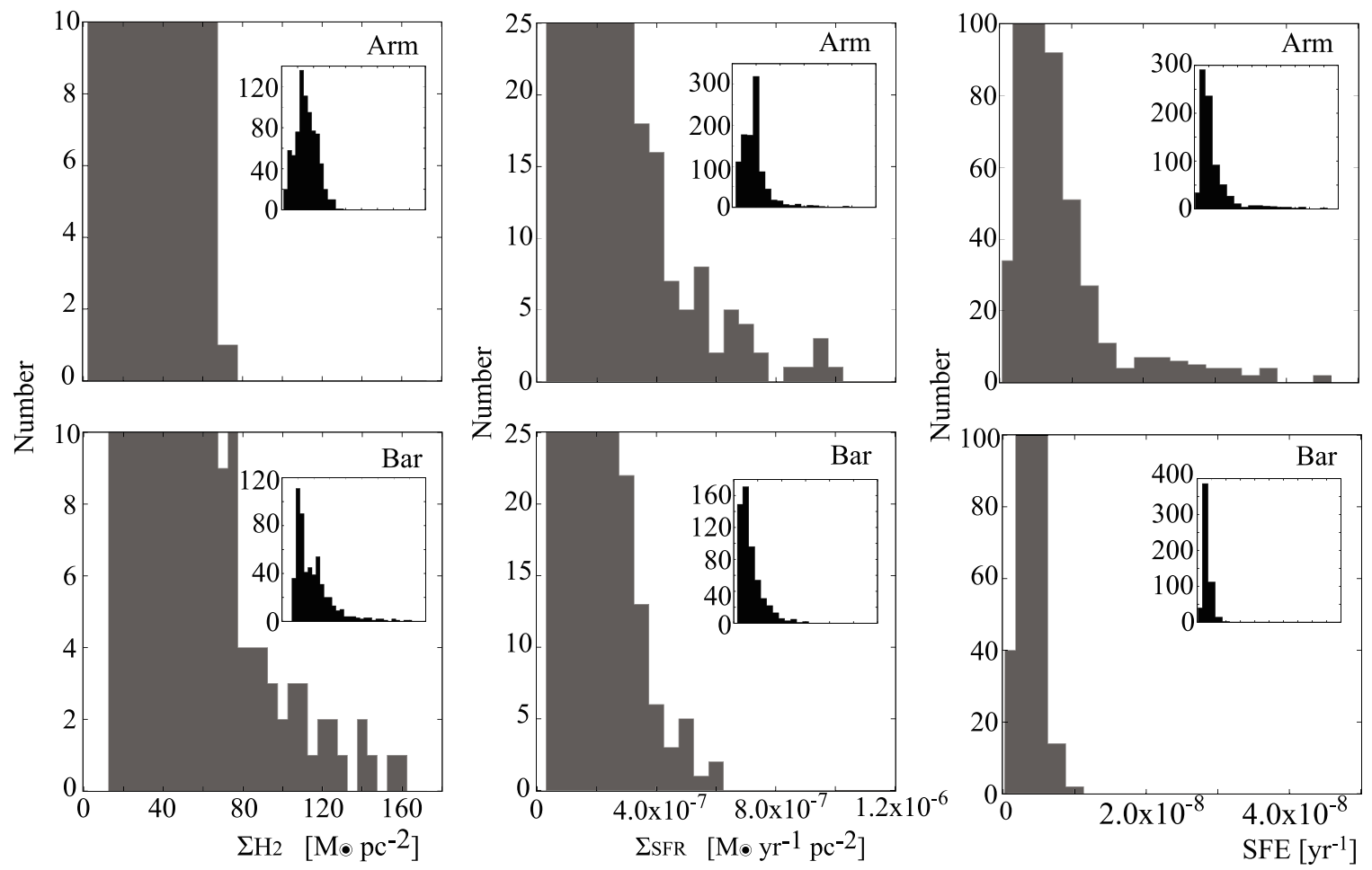

Figure 9. Histograms of (left) surface density $\Sigma_{\mathrm{H}_{2}}$, (middle) area-averaged SFR $\Sigma_{\mathrm{SFR}}$, and (right) SFE. The insets are overall view of the histograms.

are higher by a factor of 6.9 and 1.3 , respectively than those of the entire disk region.

We, however, note that NGC 4303 is suggested to harbor an active galactic nucleus (AGN) in addition to a starburst (e.g., Kennicutt et al. 1989; Colina et al. 1997; Tschöke et al. 2000), and our analysis could suffer from AGN contamination. We checked the spectral energy distribution of the nucleus in 1-24 $\mu \mathrm{m}$, using the archival data (Catalog of Infrared Observations; ${ }^{8}$ Gezari et al. 1993, 2000). We found a flat spectrum from 10 to $24 \mu \mathrm{m}$, similar to AGN spectra (Alonso-Herrero et al. 2003). Thus, $24 \mu \mathrm{m}$ emission from the nucleus is dominated by the AGN. This indicates that the much higher values of SFR in the nucleus are overestimated.

\subsection{Comments on Metallicity-dependent $X_{\mathrm{CO}}$}

We adopt the standard conversion factor $X_{\mathrm{CO}}$. However, there are some arguments supporting a metallicity-dependent $X_{\mathrm{CO}}$ (e.g., Arimoto et al. 1996; Boselli et al. 2002). NGC 4303 has a relatively large radial metallicity gradient (Skillman et al. 1996), which may affect the result that SFE is low in the bar (inner disk) and high in spiral arms (outer disk). We confirmed as follows that this does not change the results (except for the nucleus, where metallicity is exceptionally high).

The average metallicities $(12+\log [\mathrm{O} / \mathrm{H}])$ in the nucleus, bar, and spiral arms (the definition is same as in Figure 8(b)) are 9.5, 9.3, and 9.2 from Skillman et al. (1996). If we adopt the $X_{\mathrm{CO}}-$ metallicity relation of Boselli et al. (2002), the gas surface densities decrease, and the average SFEs increase (by a factor of 4.2, 2.3, and 1.8), respectively. The difference in SFE between the bar and spiral arms is reduced only by $30 \%$. This is too small to change the trend that the SFE is roughly a factor of two higher in spiral arms than in bar. A significant effect appears only in the nucleus, where a much higher metallicity results in

\footnotetext{
8 http://ircatalog.gsfc.nasa.gov/
}

a significantly higher SFE (a factor of two to four higher than that from the standard $X_{\mathrm{CO}}$ ).

\section{THE KENNICUTT-SCHMIDT LAW}

The KS law shows a tight correlation between the gas surface density $\Sigma_{\text {gas }}$ and SFR density $\Sigma_{\text {SFR }}$ (Kennicutt 1998) as we mentioned in Section 1. The difference in SFE between the bar and spiral arms may affect this correlation. Figure 10(a) shows the surface densities of gas $\left(\Sigma_{\mathrm{H}_{2}}\left[M_{\odot} \mathrm{pc}^{-2}\right]\right)$ and areaaveraged $\operatorname{SFR}\left(\Sigma_{\mathrm{SFR}}\left[M_{\odot} \mathrm{yr}^{-1} \mathrm{pc}^{-2}\right]\right)$ for our data at $\sim 500 \mathrm{pc}$ resolution. We use the same axis ranges as those in Figure 9 of Kennicutt (1998), spanning five orders of magnitude in $\Sigma_{\mathrm{H}_{2}}$ and seven orders of magnitude in $\Sigma_{\mathrm{SFR}}$, a range which covers from normal spiral galaxies to circumstellar starburst.

The central region of our data could be contaminated with AGNs, and the SFR calculated form $24 \mu \mathrm{m}$ could be overestimated. Therefore, we fit straight lines to two sets of data, i.e., with and without the central region, to see the effect of AGN on the KS fits. Figure 10(a) shows our least-square fit results: the orange and blue solid lines are for the data with and without the central region and are $\Sigma_{\mathrm{SFR}}=10^{-2.61 \pm 0.04} \times \Sigma_{\mathrm{H}_{2}}^{1.18 \pm 0.02}$ and $\Sigma_{\mathrm{SFR}}=10^{-1.78 \pm 0.05} \times \Sigma_{\mathrm{H}_{2}}^{0.67 \pm 0.03}$, respectively. The contamination of AGNs apparently increases the index $N$. The analysis of Kennicutt (1998) might have been contaminated with AGNs, which are typically found in galaxies with circumnuclear starbursts.

\subsection{Comparison with Previous Studies}

Figure 10(a) compares our result with those from previous studies. The solid black line is the result of Kennicutt (1998); $N=1.4 \pm 0.15$. They calculated gas surface densities with $\mathrm{CO}$ data (gas density), and $\mathrm{H}$ I and $\mathrm{CO}$ data (total gas density). The gray region indicates the scatter in Kennicutt (1998, their Figure 9). Dashed lines are the fit in Kennicutt et al. (2007); the 
(a)

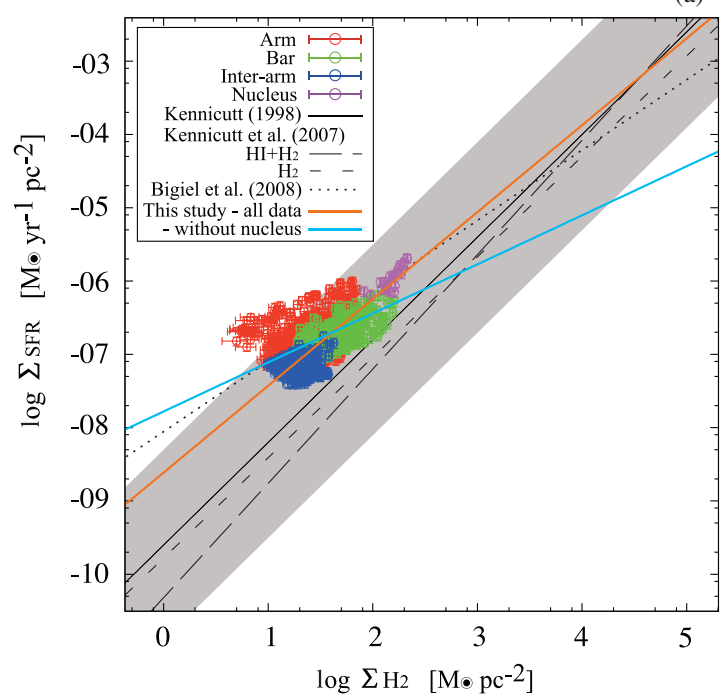

(b)

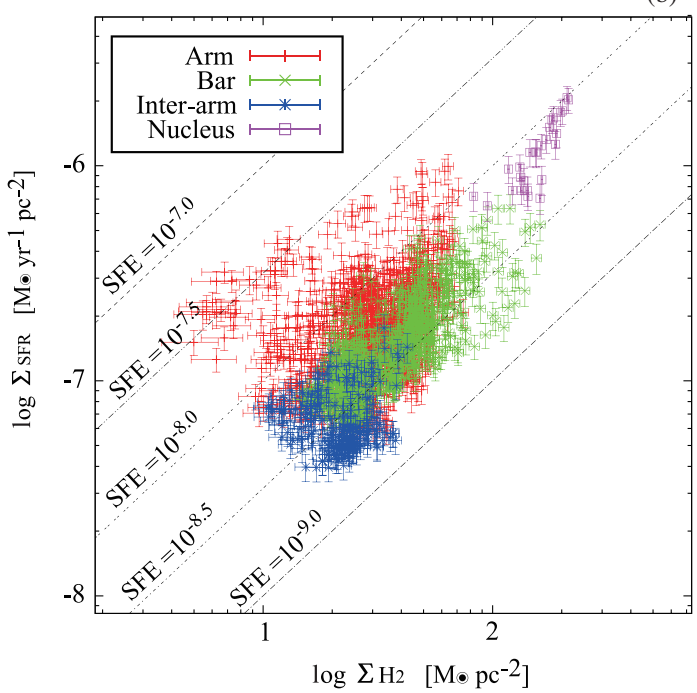

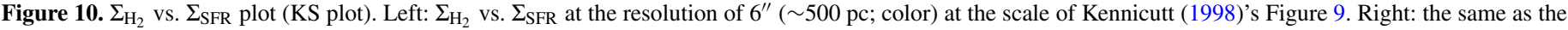
left panel, but at a larger scale.

large and small dashes are the fits for $\mathrm{H} \mathrm{I}$ and $\mathrm{CO}(N=1.56 \pm$ $0.04)$ and for CO-only $(N=1.37 \pm 0.03)$, respectively. The dotted line is from Bigiel et al. (2008) and is for $N=0.96 \pm$ 0.07 . Our result, $N=0.67$, is closer to Bigiel et al. (2008), than to Kennicutt (1998), though it is smaller than the linear correlation $N=1$.

Our data lie roughly in the range of Kennicutt (1998; gray region), although some data points deviate from the exact range. The deviations occur on the spiral arms, where the offsets between the gas and star-forming regions are large (Section 4.4). At the $500 \mathrm{pc}$ resolution, the KS law is present, but suffers slightly from the offset effect. Two types of scatter appear in Figures 10(a) and (b). One is the scatter of NGC 4303 itself, i.e., its deviation from the average of galaxies, and the other is within the disk of NGC 4303. The distribution of our points is concentrated in a small region, and the scatter within the galaxy is smaller than that of Kennicutt (1998). Therefore, the scatter among galaxies seems dominant. Overall, NGC 4303 fits on the plot of Kennicutt (1998), but has slightly higher SFE than the average.

Both $\Sigma_{\mathrm{H}_{2}}$ and $\Sigma_{\mathrm{SFR}}$ show one order of magnitude scatters in Figure 10(b), which is consistent with the results of Bigiel et al. (2008, their Figure 4). Therefore, an order of magnitude scatter of star formation activity is common at a sub-kpc scale. Leroy et al. (2008) showed that SFE varies strongly with local conditions. Since the KS law breaks down at the scale of a few $100 \mathrm{pc}$, we could attribute the variation of SFE to local environments at the scale of a few $100 \mathrm{pc}$. One of the key environmental factors might be galactic dynamics (e.g., spiral arms).

We verify a scatter of NGC 4303 itself among nearby galaxies, i.e., a scatter from the KS law. Rough estimation of scatter shows that our results are a factor of $\sim 5$ higher than that of Kennicutt (1998). However, the KS plot has a \pm 1 order of magnitude scatter, as we discussed in Section 5.1. Even though our data are the fit by Kennicutt (1998), they are still within the scatter (gray region).

\section{SUMMARY}

We observed the barred spiral galaxy NGC 4303 in the ${ }^{12} \mathrm{CO}$ $(J=1-0)$ line with NRO45 and CARMA. The combination of NRO45 and CARMA provided an unprecedented high image fidelity as well as a high angular resolution $\left(3^{\prime \prime} .2 \sim 250 \mathrm{pc}\right)$, which are critical for the accurate measurements of gas surface density and mass at high resolution. We discussed SFR and SFE quantitatively. Our results are summarized as follows.

1. CO emission is detected over the entire disk, i.e., almost everywhere including inter-arm regions and the downstream side of the bar. There are remarkable concentrations along the offset ridges of the bar and in the ring structure in the nucleus $(r \sim 1.6 \mathrm{kpc}$ area). The gas in the spiral arms extends from the end of the offset ridges toward the outer region. The surface densities in the outer spiral arms and offset ridges are similar at high resolution.

2. Spatial offsets between $\mathrm{H} \alpha$ and $\mathrm{CO}$ peaks exist along the spiral arms. $\mathrm{H} \alpha$ emission is seen at the downstream side of gas flow, while the $\mathrm{CO}$ emission is upstream of the gas flow. The delay of star formation from the formation of GMC on spiral arms would cause such offsets.

3. The azimuthal averaged SFE decreases steeply from the circumnuclear disk to the bar and increases toward the spiral arms. The comparison of SFE in the bar and spiral arms shows that SFE is about twice as high in the arms as those of in the bar.

4. Extreme $\Sigma_{\mathrm{SFR}}$ and SFE are found in the spiral arms, but not in the bar, indicating that the trigger of star formation is related not only to the amount of available gas, but also to the environment, such as galactic dynamics around spiral arms and the bar. The presence of the active star-forming regions along the spiral arms confirms the visual impression that star formation is more active in spiral arms, or reduced significantly in bar.

5. The SFE derived with a metallicity-dependent $X_{\mathrm{CO}}$ does not change the conclusion, i.e., higher SFE in the spiral arms than in the bar, since the difference between the bar and the spiral arms is reduced by only around $30 \%$. However, $\mathrm{SFE}$ in the circumnuclear regions is a factor of two to three higher than the results with the SFE derived by a standard $X_{\mathrm{CO}}$, since metallicity of the circumnuclear is significantly high.

6. The KS law appears to break down at our highest spatial resolution $(\sim 250 \mathrm{pc})$; due to the spatial offsets, we find 
between parental gas and star-forming regions. The correlation reappears if we smooth the images to $\sim 500 \mathrm{pc}$ resolution. The least-squares fit of all data in the KS law is $\Sigma_{\mathrm{SFR}}=10^{-2.61 \pm 0.04} \times \Sigma_{\mathrm{H}_{2}}^{1.18 \pm 0.02}$. NGC 4303 lies roughly in the range of Kennicutt (1998), though it has slightly higher SFE than average value among nearby galaxies.

We are very grateful to Yasutaka Kurono for helping us to combine our data, Takeshi Okuda for data analysis and discussions, Norio Ikeda for the three-dimensional FITS viewer package " $F A Z Z$," and Jennifer Donovan Meyer and James Barrett for helpful comments on the English. We thank the NRO staff for NRO45 observations, the CARMA staff and CARMA summer school 2008 participants for CARMA observations and lectures, and helpful experience. This work made use of the Spitzer Space Telescope, which is operated by the Jet Propulsion Laboratory, California Institute of Technology under a contract with the NASA, and the NASA/IPAC Extragalactic Database (NED). Support for CARMA construction was derived from the Gordon and Betty Moore Foundation, the Eileen and Kenneth Norris Foundation, the Caltech Associates, the states of California, Illinois, and Maryland, and the National Science Foundation. Ongoing CARMA development and operations are supported by the National Science Foundation under a cooperative agreement and by the CARMA partner universities. This research was partially supported by HST-AR-11261.01.

\section{REFERENCES}

Alonso-Herrero, A., Quillen, A. C., Rieke, G. H., Ivanov, V. D., \& Efstathiou, A. 2003, AJ, 126, 81

Arimoto, N., Sofue, Y., \& Tsujimoto, T. 1996, PASJ, 48, 275

Bigiel, F., Leroy, A., Walter, F., Brinks, E., de Blok, W. J. G., Madore, B., \& Thornley, M. D. 2008, AJ, 136, 2846

Boselli, A., Lequeux, J., \& Gavazzi, G. 2002, A\&A, 384, 33

Calzetti, D., et al. 2007, ApJ, 666, 870

Colina, L., Garcia Vargas, M. L., Mas-Hesse, J. M., Alberdi, A., \& Krabbe, A. 1997, ApJ, 484, L41

Corder, S. A. 2008, PhD thesis, California Institute of Technology

de Vaucouleurs, G., de Vaucouleurs, A., Corwin, H. G., Jr., Buta, R. J., Paturel, G., \& Fouqué, P. 1991, Third Reference Catalogue of Bright Galaxies (Berlin: Springer)

Downes, D., Reynaud, D., Solomon, P. M., \& Radford, S. J. E. 1996, ApJ, 461, 186

Egusa, F., Kohno, K., Sofue, Y., Nakanishi, H., \& Komugi, S. 2009, ApJ, 697, 1870

Egusa, F., Sofue, Y., \& Nakanishi, H. 2004, PASJ, 56, L45

Ferrarese, L., et al. 1996, ApJ, 464, 568
Gezari, D. Y., Pitts, P. S., \& Schmitz, M. 2000, The Catalog of Infrared Observations, 5th ed. (http://ircatalog.gsfc.nasa.gov/)

Gezari, D., Schmitz, M., Pitts, P. S., \& Mead, J. M. 1993, Far Infrared Supplement: Catalog of Infrared Observations, 3rd ed. (NASA Ref. Publ.; Greenbelt, MD: NASA Goddard Space Flight Center)

Helfer, T. T., Thornley, M. D., Regan, M. W., Wong, T., Sheth, K., Vogel, S. N. Blitz, L., \& Bock, D. C.-J. 2003, ApJS, 145, 259

Ishizuki, S. 1999, Adv. Space Res., 23, 991

Kenney, J. D. P., Carlstrom, J. E., \& Young, J. S. 1993, ApJ, 418, 687

Kennicutt, R. C., Jr. 1998, ARA\&A, 36, 189

Kennicutt, R. C., Jr., Keel, W. C., \& Blaha, C. A. 1989, AJ, 97, 1022

Kennicutt, R. C., Jr., et al. 2007, ApJ, 671, 333

Knapen, J. H., Stedman, S., Bramich, D. M., Folkes, S. L., \& Bradley, T. R. 2004, A\&A, 426, 1135

Koda, J., \& Sofue, Y. 2006, PASJ, 58, 299

Koda, J., et al. 2009, ApJ, 700, L132

Koopmann, R. A., Kenney, J. D. P., \& Young, J. 2001, ApJS, 135, 125

Kuno, N., et al. 2007, PASJ, 59, 117

Kurono, Y., Morita, K., \& Kamazaki, T. 2009, PASJ, 61, 873

Laine, S., Shlosman, I., Knapen, J. H., \& Peletier, R. F. 2002, ApJ, 567, 97

Leroy, A. K., Walter, F., Brinks, E., Bigiel, F., de Blok, W. J. G., Madore, B., \& Thornley, M. D. 2008, AJ, 136, 2782

Möllenhoff, C., \& Heidt, J. 2001, A\&A, 368, 16

Pérez-Ramírez, D., Knapen, J. H., Peletier, R. F., Laine, S., Doyon, R., \& Nadeau, D. 2000, MNRAS, 317, 234

Sakamoto, K., Okumura, S. K., Ishizuki, S., \& Scoville, N. Z. 1999a, ApJ, 525, 691

Sakamoto, K., Okumura, S. K., Ishizuki, S., \& Scoville, N. Z. 1999b, ApJS, 124,403

Sault, R. J., Teuben, P. J., \& Wright, M. C. H. 1995, in ASP Conf. Ser. 77, Astronomical Data Analysis Software and Systems IV, ed. R. A. Shaw, H E. Payne, \& J. J. E. Hayes (San Francisco, CA: ASP), 433

Sawada, T., et al. 2008, PASJ, 60, 445

Schinnerer, E., Maciejewski, W., Scoville, N., \& Moustakas, L. A. 2002, ApJ, 575,826

Schmidt, M. 1959, ApJ, 129, 243

Scoville, N. Z., \& Sanders, D. B. 1987, in Interstellar Processes, ed. D. Hollenbach \& H. Thronson (Dordrecht: Reidel), 21

Sheth, K., Regan, M. W., Vogel, S. N., \& Teuben, P. J. 2000, ApJ, 532, 221

Sheth, K., Vogel, S. N., Regan, M. W., Teuben, P. J., Harris, A. I., \& Thornley, M. D. 2002, AJ, 124, 2581

Skillman, E. D., Kennicutt, R. C., Jr., Shields, G. A., \& Zaritsky, D. 1996, ApJ, 462,147

Sorai, K., Sunada, K., Okumura, S. K., Iwasa, T., Tanaka, A., Natori, K., \& Onuki, H. 2000, SPIE, 4015, 86

Strong, A. W., et al. 1987, Proc. 20th Int. Cosmic-Ray Conf., 1, 125

Sunada, K., Yamaguchi, C., Nakai, N., Sorai, K., Okumura, S. K., \& Ukita, N. 2000, SPIE, 4015, 237

Tamburro, D., Rix, H.-W., Walter, F., Brinks, E., de Blok, W. J. G., Kennicutt, R. C., \& Mac Low, M.-M. 2008, AJ, 136, 2872

Tschöke, D., Hensler, G., \& Junkes, N. 2000, A\&A, 360, 447

Verley, S., Corbelli, E., Giovanardi, C., \& Hunt, L. K. 2010, A\&A, 510, 64

Wong, T., \& Blitz, L. 2002, ApJ, 569, 175

Young, J. S., \& Scoville, N. Z. 1991, ARA\&A, 29, 581

\footnotetext{
9 see http://hibari.isas.jaxa.jp/nikeda/fazz/fazz.html
} 